\title{
The James Webb Space Telescope: Extending the Science
}

\author{
Jonathan P. Gardner
}

NASA's Goddard Space Flight Center, Observational Cosmology Laboratory, Astrophysics Science

Division, Code 665, Greenbelt MD 20771, jonathan.p.gardner@nasa.gov

\begin{abstract}
The science objectives of the James Webb Space Telescope fall into four themes. The End of the Dark Ages: First Light and Reionization theme seeks to identify the first luminous sources to form and to determine the ionization history of the universe. The Assembly of Galaxies theme seeks to determine how galaxies and the dark matter, gas, stars, metals, morphological structures, and black holes within them evolved from the epoch of reionization to the present. The Birth of Stars and Protoplanetary Systems theme seeks to unravel the birth and early evolution of stars, from infall onto dustenshrouded protostars, to the genesis of planetary systems. The Planetary Systems and the Origins of Life theme seeks to determine the physical and chemical properties of planetary systems around nearby stars and of our own, and to investigate the potential for life in those systems. These four science themes were used to establish the design requirements for the observatory and instrumentation. Since Webb's capabilities are unique, those science themes will remain relevant through launch and operations and goals contained within these themes will continue to guide the design and implementation choices for the mission. More recently, it has also become clear that Webb will make major contributions to other areas of research, including dark energy, dark matter, exoplanet characterization and Solar System objects. In this paper, I review the original four science themes and discuss how the scientific output of Webb will extend to these new areas of research.
\end{abstract}

Keywords: Space Telescopes, Science Objectives, Exoplanets, Dark Matter, Dark Energy

\section{INTRODUCTION}

The James Webb Space Telescope (JWST) is a 6.6m space telescope that will be launched in 2018. It is the successor to the Hubble and Spitzer Space Telescopes and like those missions will conduct a wide-ranging science program, selected through annual calls for peer-reviewed proposals. JWST will impact nearly every science topic within astronomy, from Solar System observations to Cosmology.

In order to guide the design and construction of the observatory, the JWST Science Working Group organized the science goals of JWST along four themes, and from those themes derived the science requirements. A description of those science goals and the derived design was published in Gardner et al. 2006. Since that time, there have been additional efforts to explore the science potential of JWST. The Science Working Group has developed a series of white papers on various topics including exoplanets, resolved stellar populations, dark energy and Solar System objects. There are numerous papers in the refereed literature that reference JWST. In addition, there have been two conferences which specifically address the JWST science goals; Astrophysics in the Next Decade, The James Webb Space Telescope and Concurrent Facilities, (Thronson, Stiavelli \& Tielens, 2007), and Frontier Science Opportunities with JWST, (http://www.stsci.edu/institute/conference/jwst2011).

In this paper I review the original science goals of JWST (Section 2) and describe recent work on dark energy (Section 3), dark matter (Section 4), exoplanet characterization (Section 5) and Solar System objects (Section 6).

\section{JWST'S SCIENCE GOALS}

The original science goals for JWST are organized into four themes. The End of the Dark Ages: First Light and Reionization theme seeks to identify the first luminous sources to form and to determine the ionization history of the universe. The Assembly of Galaxies theme seeks to determine how galaxies and the dark matter, gas, stars, metals, morphological structures, and black holes within them evolved from the epoch of reionization to the present. The Birth of Stars and Protoplanetary Systems theme seeks to unravel the birth and early evolution of stars, from infall onto dustenshrouded protostars, to the genesis of planetary systems. The Planetary Systems and the Origins of Life theme seeks to 
determine the physical and chemical properties of planetary systems around nearby stars and of our own, and to investigate the potential for life in those systems.

The End of the Dark Ages: First Light and Reionization: The Big Bang determined the initial distribution of energy and matter in the Universe, including dark energy, dark matter, ordinary matter, neutrinos and radiation. The release of the Cosmic Microwave Background marked the formation of Hydrogen atoms from protons and electrons. Sometime between 100 and 400 million years after the Big Bang, the first stars and galaxies started to form. Although the overall density of matter in the Universe decreases with the cosmic expansion, the density of individual structures, called dark matter halos, increases through gravitational attraction. When the dark matter halos reached a size large enough to enable the baryonic gas within them to dissipate their energy through molecular cooling, the first stars formed. These stars, however, likely disrupted any molecular Hydrogen in the surrounding area and did not form into galaxies. The first stars were likely to be very massive and evolve quickly to end their lives as Supernova or through direct collapse into black holes. JWST might be able to detect these Supernova; they should be bright enough to see and could be identified as Lyman alpha dropouts at very high redshift. Current theory does not make robust predictions about the frequency, however, so it is difficult to know if they were common enough for JWST to find in a reasonable amount of observing time.

When the dark matter halos became large enough to enable atomic cooling of the baryons, the first galaxies formed. These first galaxies are expected to have about $10^{6}$ Solar masses in stars, and be visible at $\mathrm{AB}$ magnitudes between 30 and 31, making them detectable in a JWST ultra-deep field.

The first galaxies put out ultraviolet radiation and began to create ionized bubbles in inter-galactic medium. The bubbles got bigger and joined together, a process that completed by about 1 billion years after the Big Bang, a redshift of 6 . Using spectroscopy of the brightest high-redshift sources known, JWST can observe the process of Reionization. In addition, JWST will test theories that predict that Reionization has an effect on the formation of galaxies.

The Assembly of Galaxies: Galaxies grew through a hierarchical buildup of small dark matter halos into larger structures. Baryonic star formation could be triggered during the mergers and also could proceed through more passive secular evolution. The stars within a galaxy could have a range of ages; the oldest stars could have formed in situ, or in a dwarf galaxy that subsequently merged into the larger galaxy. Because of the wide diversity of galaxies in morphology, stellar populations, star-formation rates and metallicity, large samples are needed.

Uring the H $\alpha$ line, JWST can measure star-formation rates as low as 1 Solar mass per year at $\mathrm{z} \sim 5$. The existence of old stellar populations, revealed in rest-frame near-infrared observations, and the emergence of red quiescent galaxies in which star-formation has ceased, provide important clues on the end states of galaxy evolution. Metallicity, a measure of the heavy elements formed in stars, reveals one of two cosmologically significant sources of luminosity in the Universe, along with gravitational accretion energy.

Despite the variety of galaxy properties observed today, galaxies obey a number of remarkably tight scaling relations between basic properties of luminosity, size, and kinematics and metal enrichment. These include the Tully-Fisher relation for disk galaxies, the "fundamental plane" and its projections for spheroids, and a correlation between the mass of the central black hole and the properties of the surrounding spheroid. By measuring these parameters out to high redshift, JWST can determine the evolutionary processes that created them and address the relationship between the dark matter and the stars in galaxies. By studying starbursting galaxies and active galactic nuclei, JWST can examine galaxy evolution under the most extreme conditions.

The Birth of Stars and Protoplanetary Systems: Within our own galaxy, stars form in small regions undergoing gravitational collapse within larger molecular clouds. With high spatial resolution imaging and spectroscopy in the midinfrared, JWST will be able to penetrate the dusty clouds where stars form to investigate the roles of magnetic fields, turbulence and rotation in this process. The dynamics of protostellar collapse can be diagnosed through mid-infrared imaging spectroscopy of shocks.

The formation of massive stars (through a process not yet well understood) produces intense winds and ionizing radiation which impacts the surrounding molecular cloud material and the nascent circumstellar disks of adjacent low- 
mass stars. Once born, massive stars can be disturbing neighbors. Ionizing photons and strong winds from $O$ and $B$ stars damage their environment. They can destroy disks around young low-mass stars, or evaporate and compress surrounding molecular material, thus either halting or triggering further star formation in surrounding molecular material. Imaging and spectroscopic surveys will be made of dark clouds and elephant trunks in regions with recent massive star formation, to reveal populations of young stars and study their properties. By examining the masses and ages of the sources as a function of their distance from the ionization front, it will be possible to determine whether $\mathrm{O}$ and $\mathrm{B}$ stars simply reveal pre-existing star formation or trigger it directly.

The initial mass function for stars is a key product of the process of star formation, but is not well characterized at the smallest sub-stellar masses, less than 10 Jupiter masses. The relationship between free-floating objects of these masses and true planets is also unknown.

Circumstellar disks around young low-mass stars are both a product and a mediator of the star formation process, as well as the progenitors of planetary systems. High spatial resolution imaging with JWST of a large sample of silhouette disks will indicate the sizes of their particles and reveal the processes for forming planets. These planets include the heavy. elements, carbon, nitrogen and oxygen, which make up life on Earth. In cold quiescent cloud cores, gas-phase molecules stick to dust grains, forming icy mantles on silicate cores. When the heavy elements are frozen out, the chemical composition is initially modified via grain-surface reactions. Then, as a central protostar develops and heats up its envelope, the ices evaporate back to the gas phase and a rich mixture of organic compounds develops, subject to further processing by UV radiation, X-rays and thermal cycles.

Planetary Systems and the Origins of Life: Understanding the origin of the Earth and its ability to support life is an important objective for astronomy. Key parts of the story include understanding the formation of small objects and how they combine to form large ones, learning how they reach their present orbits, learning how the large planets affect the others in systems like ours, and learning about the chemical and physical history of the small and large objects that formed the Earth and delivered the necessary chemical precursors for life. The cool objects and dust in the outer Solar System are evidence of conditions in the early Solar System, and are directly comparable to cool objects and dust observed around other stars.

The remnant of the circumstellar disk that formed our Solar System is observable today as the smaller planets, moons, asteroids and comets, along with the interplanetary gas. Spectroscopy and photometry of small Solar System bodies, particularly comets, at wavelengths and sensitivities unavailable from the ground can identify isotopic ratios and molecular and elemental abundances. These can be compared with remnant and planet-forming disks, providing direct measurements of the smaller components of circumstellar disk formation.

Some geochemical evidence suggests that Earth's water did not originate within locally formed planetesimals at $1 \mathrm{AU}$, but the source of water is uncertain. Asteroids and comets remain a highly plausible source of Earth's organics, and the inventory of organics derived from high sensitivity infrared spectra will constrain this part of the story. JWST measurements of the composition and structure in protoplanetary disks around other stars will extend the quantification of the source of water and organics to putative habitable worlds around stars other than the Sun.

Extending the JWST Science Goals: In this section, I have reviewed the original science goals for JWST, as described by Gardner et al. 2006. It was recognized that an observatory designed to enable these goals will also be broadly capable of addressing other topics in Astronomy and that advancements in the field will make some of these other topics more compelling as time goes on. JWST will be a general user facility, with more than $80 \%$ of the observing time allocated in annual peer-reviewed calls for proposals. In the next few sections I review some recent work in several areas that have extended the JWST science goals, using the capabilities of the observatory.

\section{DARK ENERGY}

Dark energy makes up $73 \%$ of the mass-energy density of the universe and causes its expansion to accelerate. It was first discovered through studies using $\mathrm{z}<1$ type 1a supernova (SNIa) as standard candles (Riess et al. 1998; Perlmutter et al. 1999), and confirmed by evidence of deceleration at $z=1.8$, seen with the Hubble Space Telescope (HST) (Riess et al. 2001). The effects of dark energy are subtle and studying dark energy requires wide-area surveys, either to find and 
identify type Ia supernovae (SNIa) as they explode, or to obtain the large statistical datasets needed for weak lensing (WL), baryon oscillation (BAO) or cluster studies (CL). Dark energy is parameterized by evolution in the equation of state with redshift, w(z). The cosmological constant model predicts that $\mathrm{w}$ is constant. JWST was originally conceived and designed to conduct deep surveys efficiently; it is similar to the Hubble Space Telescope in its ability to conduct wide-area surveys, with a much lower survey mapping efficiency than missions like Spitzer, GALEX and WISE, which were optimized for efficient sky mapping.

Despite its small field of view, HST has contributed significantly to studies of dark energy in several ways. The GOODS project and associated follow-up discovered $21 \mathrm{SNIa}$, expanding on this result (Riess et al. 2007), and the CANDELS project is continuing these studies (Grogin et al. 2011). HST has also been used to constrain cosmological parameters and dark energy through weak lensing measurements in the COSMOS survey (Massey et al 2007) and strong gravitational lensing with measured time delays (Suyu et al 2010). NASA is studying the Wide-Field Infrared Survey Telescope (WFIRST) mission, which will constrain dark energy using SNIa, WL and BAO. ESA is studying the Euclid mission, which will constrain dark energy using WL and BAO. JWST will study dark energy in several ways, either in partnership with a dedicated wide-area mission, or on its own.

JWST studies of dark energy using type Ia supernova: A supernova search similar to that done with Hubble on GOODS would be feasible with JWST. Hubble imaged the 320 square arcmin in two GOODS fields 19 times (1 reference epoch and 18 search epochs.) The SN rate of discovery was about 1 supernova at $\mathrm{z}>1$ per epoch imaged, equivalent to $10 \mathrm{SNe}$ per square degree per epoch. With its greater sensitivity, JWST could reach the GOODS depth in 30 s of exposure time, allowing a larger area to be surveyed. Alternatively, longer JWST exposures could reach to higher redshift.

Riess \& Livio (2006) have argued that there would be a particular merit in observing supernovae at $z>1.5$ in order to measure or place upper limits to supernova evolutionary or inter-galactic medium effects that might represent the limiting factor in future supernova searches. By observing SNIa in the redshift range 1.5-3 one can focus on evolutionary effects since at these redshifts the dark-energy dependent effects are negligible. The SNIa rates at these redshifts are unknown and one needs to consider a range of models assuming different values for the Type Ia delay, the time between initial population of stars form and the formation of SNIa. Longer delays mean that SNIa might be rarer at the higher redshifts and more area or more epochs would be needed to find the same number of SNIa. In addition to going to higher redshift, JWST observations will allow the measurement of rest-frame near-infrared light curves (Wood-Vasey et al 2008; Freedman et al 2009.) The effects of extinction by dust are lower in the infrared, and the peak magnitudes are standard candles at the $15 \%$ level, even without correcting for light-curve shape.

Supernova follow-up includes measuring the light curve and obtaining a spectroscopic confirmation for the redshift. In addition to following-up its own SNIa discoveries, JWST would also be able to follow-up supernovae found by other surveys. WFIRST will find hundreds to thousands of SNIa, obtain spectroscopic redshifts and follow their light curves. SNIa at $\mathrm{z} \sim 1.5$ are bright enough for JWST to obtain a spectroscopic redshift in a relatively short amount of time. If we wished for JWST to be the WFIRST supernova spectrograph, it would need to be available throughout the WFIRST SNIa observations to take spectra as targets of opportunity. Even if a full replacement of the WFIRST SNIa spectroscopy is not possible, the use of JWST to obtain spectra of a sub-set of the WFIRST SNe, such as those at the high redshift end, is a strong possibility.

JWST studies of dark energy using weak lensing: HST has measured weak lensing in the Cosmic Evolution Survey (COSMOS; Scoville et al 2007), a contiguous square field covering 1.64 square degrees. Traditional two-dimensional analysis of a weak lensing field measures the two-point correlation function of the projected weak lensing shear field. Three-dimensional analysis uses photometric redshifts to divide the galaxies into redshift bins and can constrain the growth of structure. Two-dimensional analysis of the COSMOS field has been done by Massey et al (2007) and an extension to three dimensions was done by Schrabback et al (2009).

As JWST's mapping efficiency is within a factor of a few of HST's (Gardner et al 2010), and therefore it could only do a few square degrees in a reasonable amount of observing time; JWST would not be able to do the 10,000 square degree weak lensing survey envisioned for WFIRST or Euclid. By reaching greater depth, JWST could make progress relative 
to the HST surveys by doing cosmic shear tomography at redshifts where the effects of dark energy is weak in comparison to the effects of dark matter to reveal unknown systematics in measurements made at lower redshift.

As with the SNIa, JWST could contribute to the spectroscopy needed by a wide-area survey mission. Weak lensing studies of dark energy need spectroscopic redshifts to calibrate the photometric redshifts used in three-dimensional tomography. Gehrels et al (2009) argue that 30,000 to 100,000 spectroscopic redshifts within 1 square degree are needed. Using the multi-object spectrograph in NIRSpec at R 100, JWST could do this with about 1000 hours of observing time.

JWST contributions to measurements of $\mathrm{H}_{0}$ : While the equation of state of the dark energy is investigated by measuring the expansion rate as a function of redshitt $\mathrm{H}(\mathrm{z})$, the measurements are anchored in the current value of the Hubble constant, $\mathrm{H}_{0}$. A given error on the Hubble constant leads to twice that error on the measurement of $\mathrm{w}$. JWST might be able to achieve a $1 \%$ measurement of the Hubble constant, which would imply a $2 \%$ measurement of w. Riess et al (2011) used HST to build a distance ladder that constrains $\mathrm{H}_{0}$ to $3 \%$; further refinements with HST and JWST are possible.

Measurements of $\mathrm{H}_{0}$ require a distance ladder to connect nearby sources, whose distances are measured through geometric means including parallax, to galaxies participating in the Hubble expansion at distances $>100 \mathrm{Mpc}$. Riess et al (2011) anchor their measurements in a water maser in Keplerian orbit around a supermassive black hole in the galaxy NGC4258 (e.g. Humphreys et al 2008), which gives a geometric distance. By identifying Cepheids in that galaxy, and Cepheids in the host galaxies of nearby SNIa they can tie the distances together. In addition, future observations by the European GAIA astrometry mission will measure parallax distances to hundreds of Milky Way Cepheids.

JWST will be able to extend this technique to achieve a $1 \%$ measurement of $\mathrm{H}_{0}$. By reaching deeper, JWST can measure Cepheid distances to more distant SNIa host galaxies, extending the volume. In addition, between now and JWST science operations, there will be more SNIa in nearby galaxies, so the sample size available for JWST Cepheid measurements will increase. Combining GAIA results with the possible discovery of additional maser galaxies will further reduce the errors. Measurements of $\mathrm{H}_{0}$ can be competitive with other methods of constraining the current equation of state of the dark energy, $w_{0}$. If $w_{0}$ is shown to be significantly different from -1 , that would be evidence against the cosmological constant model.

Other JWST studies of dark energy: JWST can also study dark energy in other ways. Using the multi-object spectroscopic capability of NIRSpec, JWST can measure the velocity dispersions of any know massive cluster of galaxies, constraining the mass of the clusters, and therefore dark energy through its effect on the growth of structure.

A strong gravitational lens system with measured time delays between multiple images provides a measure of the angular diameter distances to the lens, to the source and between the lens and source. The technique is most sensitive to HO, but also depends on other cosmological parameters. . The results depend on the distribution of mass in the lens, and therefore are model dependent, although the mass distribution of the lens can be constrained by weak lensing analysis or stellar kinematics in the lens galaxy. Recently, Suyu et al (2010) measured time delays in the lens system B1608+656 and constrained $\mathrm{HO}$ to about $4 \%$. Their constraints on the equation of state of the dark energy are comparable to current constraints from the BAO method (under the assumption of a flat geometry). Strong lensing of time-variable sources are rare, but the Large Synoptic Survey Telescope might find many examples (Marshall et al. 2010).

\section{DARK MATTER}

Dark matter is made up of a particle that interacts with itself and with baryonic matter only through gravity. It makes up $84 \%$ of the matter and $23 \%$ of the mass-energy density of the universe (Jarosik et al 2011). The effects of dark matter are seen in galaxy rotation curves, the dynamics of galaxy clusters and through gravitational lensing. The standard theory of structure formation in the universe is based on cold dark matter. The dark matter falls into primordial overdensities which build up over time. When the dark matter halos are large enough to enable the baryons to dissipate through molecular cooling, the first stars form at redshifts $z \sim 30$. Those first stars are probably very massive and explode as supernovae, disrupting the local environment. When the dark matter haloes become large enough for atomic cooling, then the first galaxies will form, at $z \sim 15$. 

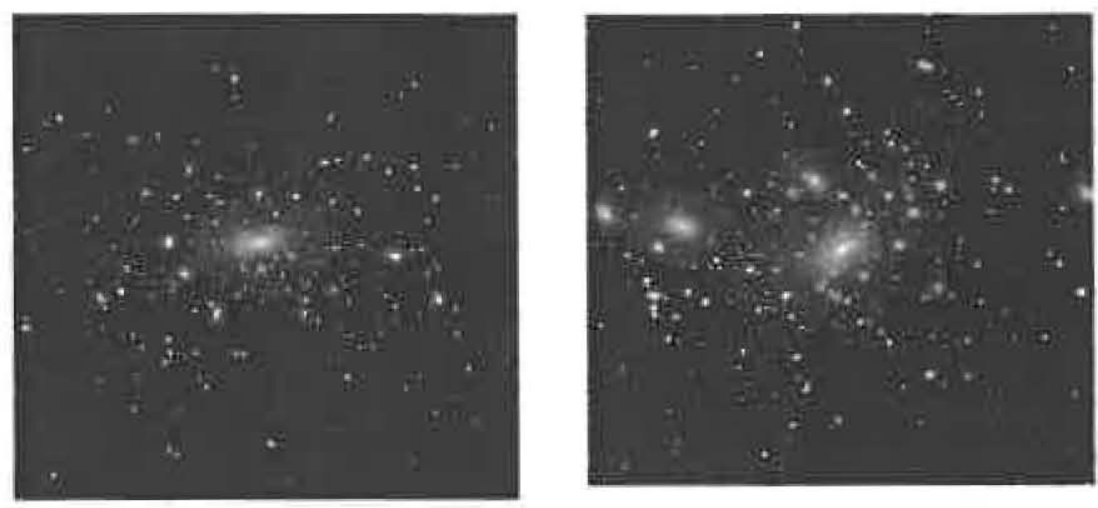

Theory
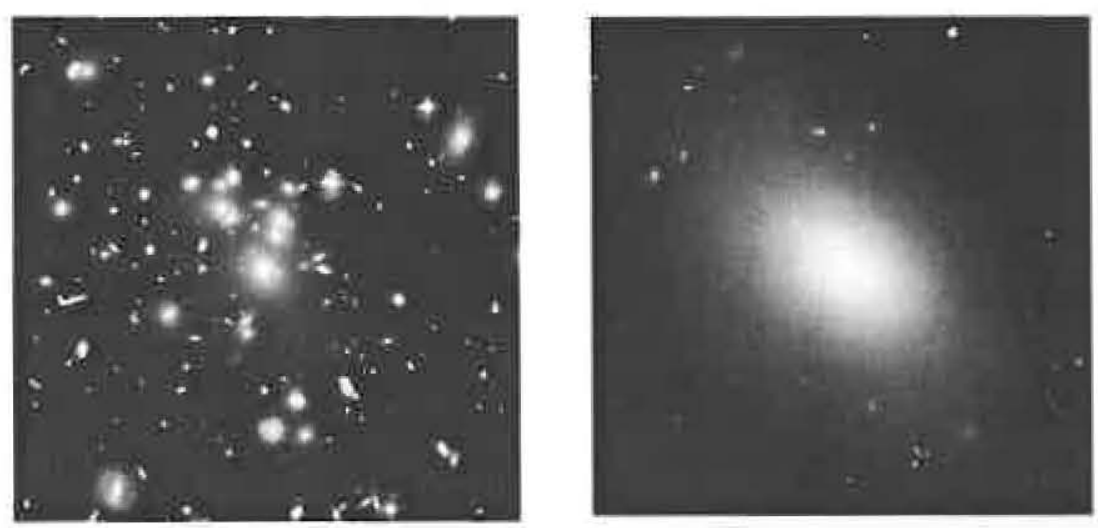

Observation

Figure 1. Top: A simulation of the dark matter in a galaxy cluster (left) and in a galaxy (right) (Kravtsov, 2010). Bottom: An observed cluster (left) and an observed galaxy. In the simulation of the dark matter, the cluster and galaxy are self-similar, with lots of substructure at all scales. In the observations of the stars, there is little substructure at the dwarf galaxy scale.

The dark matter haloes grow through merging, and the result is a distribution of halo masses. The gravity-based models predict far more small dark matter haloes than there are satellite galaxies observed (Figure 1). For example, models of typical $\mathrm{M}^{*}$ galaxies predict hundreds of small dark matter halo companions, while there are typically only a few satellite galaxies observed. There are two possibilities: either the dark matter halos exist but do not form stars, or there is a problem with the theory. Feedback by early supernovae could result in dark matter halos that are devoid of stars, while non-gravitational self-interaction by the dark matter could result in a different distribution of halo masses. Which explanation connects the theory with the observations is one of the profound open questions in dark matter research.

Gravitational lensing is one of the best ways of investigating dark matter, since it is sensitive only to the total mass and its distribution along the line of sight. Weak lensing bas been used to map the dark matter distribution in clusters of galaxies and statistically in the universe. Strong lensing, in which the light from a background quasar is lensed by a foreground galaxy into multiple images, can be used to investigate the mass distribution of dark matter at galaxy scales and smaller, particularly when the background quasar is variable. As the variability follows different light paths through the lens, substructure in the dark matter distribution is revealed.

There are three characteristic scales for lensing by a galaxy (Treu, 2010; figure 2). The mass associated with the galaxy itself produces an Einstein ring with a radius of order an arcsecond. Dwarf satellites would produce an Einstein ring with a radius of order a milli-arcsecond and individual stars in the galaxy produce micro-lensing on micro-arcsecond scales. These characteristic scales depend on the lensed object being a point source on those scales. If the lensed object is milliareseconds in size, then it will not be subject to micro-lensing by individual stars. It is not necessary to resolve the lens at milli- or micro-arcsecond scales; lensing by dwarf satellites or micro-lensing is revealed through anomalies in the flux ratios or time delays relative to a modeled smooth distribution of mass, such as an isothermal ellipsoid. 


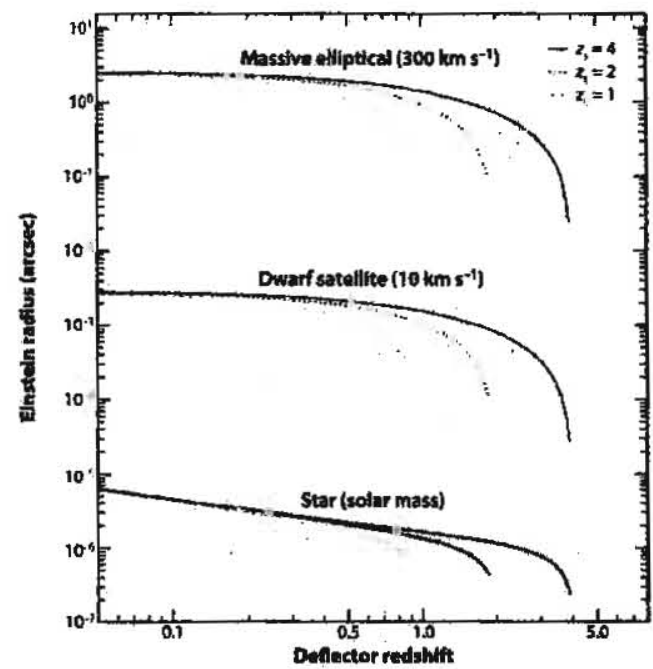

Figure 2. The Einstein radius for three characteristic scales in a lensing elliptical galaxy (Treu 2010). The galaxy itself has an Einstein radius with a size scale of an arcsecond. Dwarf satellites cause features on milli-arcsecond scales and individual stars cause micro-lensing on micro-arcsecond scales. Each size scale lenses source objects that are point sources on the same scale; so dwarf satellites, which are resolved on milli-arcsecond scales, are not subject to micro-lensing.
Characteristic scales in the source can pick out the characteristic scales in a galaxy. When the quasar accretion disk is imaged through a lensing galaxy, the accretion disk is so small that it is a point source even on micro-arcsecond scales. It is not possible to tell if a flux ratio anomaly is due to micro-lensing by individual stars, or by milli-lensing by dwarf satellites. However, the dusty torus, emitting in the mid-infrared is not affected by the micro-lensing and picks out dwarf satellite scales in the dark matter distribution. The narrow-line region is lensed by galaxy as a whole.

With its high angular resolution and extreme sensitivity in the midinfrared, JWST will be ideal to address this problem. The technique has been used with Spitzer to show that the lensed quasar, QSO $2237+0305$ has an dusty torus, consistent with the standard model of AGN. (Agol et al 2009.) Although hampered by the poor spatial resolution of Spitzer, the flux ratio anomalies were wavelengthdependent, showing that the mid-infrared light was coming from a larger region than the shorter wavelengths.

\section{EXOPLANETS}

The field of exoplanet discovery and characterization has benefitted from a wealth of data in the last few years, and tremendous progress has been made since the original publication of the JWST science goals in Gardner et al (2006.) In particular, the study of transiting exoplanets has enabled measurements of the mass, radius (e.g. Charbonneau et al 2007) and atmospheric composition (e.g. Swain et al 2008) by HST, Spitzer and other facilities. It is clear that JWST will continue to make progress in these areas. With collecting area larger than Hubble and Spitzer, JWST will be able to extend these studies to intermediate and low-mass planets, including hot Neptunes and super-Earths.

Photometric observations of transits in the near and mid-infrared with JWST offers the opportunity to obtain high precision light curves of primary and secondary eclipses of $1 R_{\text {Earrh }}$ terrestrial planets around main sequence stars. High precision light curves allow the determination of mass, radius and orbital characteristics, detection of thermal radiation from the planet (Knutson et al 2007), and the ability to search for unseen planets and moons through transit timing (Steffen et al 2007). Filter photometry could also be used to pick out atmospheric features such as the 15-micron band of $\mathrm{CO}_{2}$ (Deming et al 2009), in the absence of spectroscopic information.

Figure 3 makes it clear that spectroscopic observations with NIRSpec and MIRI will enable major advances in exoplanet characterization. The plot shows a model spectrum of HD189733b, with the wavelength coverage of the JWST spectroscopic modes labeled. Two techniques can be used to study exoplanet atmospheres. The absorption spectrum of the planet is revealed as the light from the primary star passes through the atmosphere of the planet. The emission spectrum of the planet is revealed during the secondary eclipse as the planet passes behind the star. Gas giant planets have molecular features in the atmospheric absorption spectrum, including $\mathrm{H} 2, \mathrm{CO}, \mathrm{H} 2 \mathrm{O}$ and $\mathrm{CH} 4$, and atomic features such as $\mathrm{Na}$ and $\mathrm{K}$.

For smaller planets, JWST holds the possibility of detecting water on a super-Earth in the habitable zone, although this depends on the existence and detection of the right target. Deming et al (2009) and Ricker et al (2010) describe the Transiting Exoplanet Survey Satellite (TESS) an all-sky survey mission designed to find transiting planets around nearby stars. Transits of nearby M-dwarf stars enable higher signal-to-noise measurements as the ratio of the planet size to the star size is higher. Figure 4 is a simulation of the detection of water on a super-Earth in the habitable zone. 


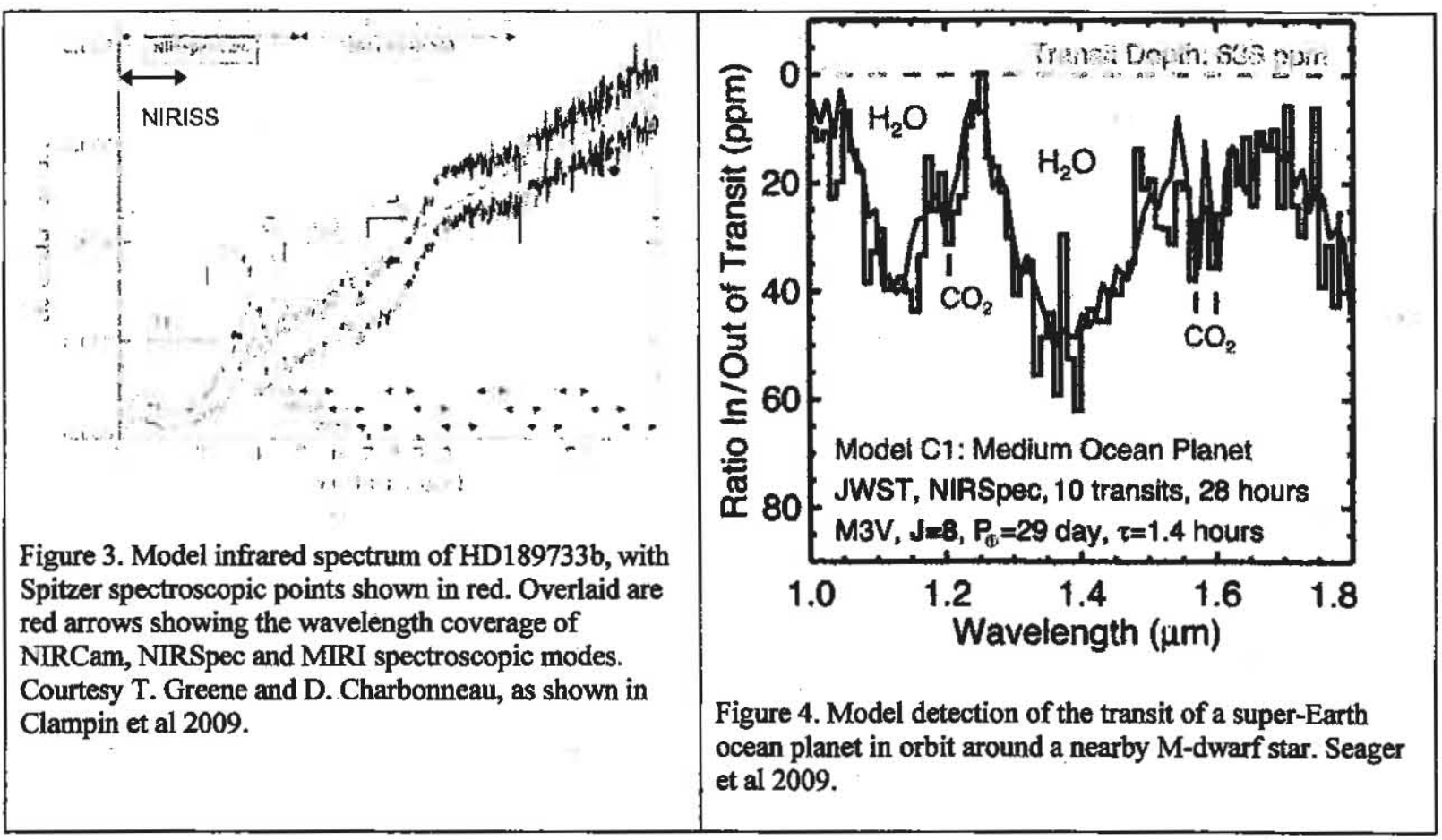

\section{SOLAR SYSTEM OBJECTS}

With the ability to track moving targets up to 30 milli-arcsec per second, JWST will be capable of observing objects in the orbit of Mars and further. It will provide spatial resolutions of $66 \mathrm{~km}$ on Mars, $230 \mathrm{~km}$ on Jupiter and its moons, 420 $\mathrm{km}$ on Saturn and its moons and approximately $1500 \mathrm{~km}$ on Pluto or Neptune. Since the original JWST science goals were published by Gardner et al 2006, there has been a considerable amount of additional work in developing Solar System science (Lunine et al 2010). In this section, I list a few representative examples.

Kuiper Belt Objects (KBOs): JWST will have the ability to obtain $R=100$ near-IR spectra and 20 to $25 \mu \mathrm{m}$ mid-IR photometry of any of the currently known KBOs. The small KBOs are the remnants of the icy building blocks that formed the giant planets and are analogous to the material in debris disks that are now being observed around other stars. Understanding the physical properties of these objects is key for determining the origins and subsequent evolution of the objects in our solar system and in other planetary systems.

Many KBOs are in binary systems which enables the mass of the system to be determined. Size information from JWST mid-IR photometry combined with the mass can be used to determine densities, a key physical property related to composition and porosity. In addition, near-IR spectroscopy of each object within a binary will determine if the compositions of the objects are indeed alike as is indicated by their similar visible colors (Benecchi et al 2009). If the surfaces of each pair within a binary are identical, it indicates that surface composition is likely primordial and provides a powerful constraint on the initial disk compositional architecture. Near-IR spectroscopy and mid-IR photometry with JWST will likely reveal additional information about large-scale collisions in the Kuiper Belt.

JWST will also allow for the detailed examination of the physical properties of a recently-discovered new population of objects with orbits that are detached from the Kuiper Belt, including Sedna.

Enceladus and Titan: JWST may be able to monitor the active hot spots of Enceladus's southern hemisphere when it comes back into view of the Earth in the year 2025. At 11 microns wavelength, the peak brightness of the hot spots, the so-called "tiger stripes" (fractures that are hot and from which the jets making up the plume are emitted) will be 100 times brighter than the MIRI limit. However the combination of reflected sunlight and thermal emission from the sun- 
warmed surface is expected to be several times brighter than the tiger stripes, so extraction of the tiger stripe signal will be challenging. Special geometries, in which eclipses by Saturn remove the reflected component, might enable these observations, or it might be easier to separate the signal at longer wavelengths.

If the tiger stripe signal can be extracted, it will be a valuable observation: By measuring the brightness of the active regions in the thermal infrared as much as a decade after Cassini's measurements, JWST will test the stability of the active area of Enceladus, which has surprised scientists with the 15 gigawatts of power (more than twice all the hot springs in Yellowstone) that it emits.

JWST can make NIRCam images, and NIRSpec spectra of Titan to build on the 2004-2017 Cassini mission survey, creating a long $(10$ year +$)$ baseline of spaceborne near-infrared observations of Titan's surface and atmosphere during a seasonal configuration hitherto unexplored in the infrared. While Cassini gets better spatial resolution, JWST will achieve higher spectral resolution with useful spatial resolution over the mid-latitude regions of Titan. Of interest is whether surface changes or secular atmospheric changes are in evidence over a decade-long timescale. With NIRSpec, the ability to probe the atmosphere over several levels down to the surface provides a unique longterm capability that is unavailable from Hubble and will cease to be available at the end of the Cassini mission. Thus JWST provides Iong-time baseline continuity throughout the infrared.

Comets: Comets are remnants of Solar System formation, and their current composition and physical properties provide a constraint on the conditions in the solar nebula 4.6 billion years ago. Observations of comets with JWST will enable investigations of the chemical composition of cometary ice and dust with unprecedented sensitivity. Near- and mid IR spectroscopy of cometary comae can be used to measure abundances of $\mathrm{H}_{2} \mathrm{O}, \mathrm{CO}, \mathrm{CO}_{2}$, and $\mathrm{CH}_{3} \mathrm{OH}$ in even relatively faint comets. Near-IR spectrometry with $\mathrm{R} \sim 1000$ resolution will be used to measure the ratio of ortho-to-para $\mathrm{H}_{2} \mathrm{O}$ (OPR) separately, possibly providing an indication of the comet's formation temperature. Likewise, mid-IR spectroscopy can determine the mineralogy of cometary dust grains. Finally, JWST's ability to image cometary nuclei at both mid and near-infrared wavelengths with high spatial resolution and sensitivity will allow high accuracy measurements of sizes and albedos of cometary nuclei. The results from cometary programs can be combined with those from programs investigating circumstellar disks and star formation regions, placing our Solar System within a complete picture of planetary system formation and evolution.

Uranus and Neptune: The outer two planets are good candidates for JWST. Their atmospheres, which are rich in hydrocarbons and seasonally varying clouds and storms, can be probed by JWST to explore their chemistry and thermal balance with unprecedented precision. Spatially resolved measurements of methane and ethane emission features in the upper atmospheres of Uranus and Neptune will help constrain the abundances of these compounds and the photochemistry involved in their formation. The abundances and variability of chemical species, including the confirmation of radicals thought to be involved in the hydrocarbon photolysis process, may be observed with JWST to more accurately characterize the atmospheric processes and formation of these planets JWST will be capable of detecting the variability of trace elements with time and altitude, the temporal variability of clouds, and will provide high-resolution images of current poorly resolved features.

Jupiter, Saturn and their Rings: Special provision of a subarray observing strategy may allow observation of Jupiter despite its large surface brightnesses, allowing for detailed observation of transient phenomena including large scale storms, aurora (in the near infrared $\mathrm{H}_{3}+$ band) and impact-generation disturbances. Saturn can be observed in all MIRI filters, across the 5 to 26 micron infrared range, allowing monitoring of seasonal changes in the high northern latitudes (which will be pointed toward Earth early in the JWST mission), and transient phenomena as for Jupiter.

JWST's spatial resolution at Saturn's rings will be a few hundred $\mathrm{km}$ in the mid infrared, comparable to the composite infrared spectrometer (CIRS) on the Cassini spacecraft currently in orbit around Saturn. CIRS is a long-slit spectrometer and requires time-intensive scanning to make an image. JWST's direct infrared imaging capability will be much more efficient than CIRS.

JWST could also cover the wavelength gap between CIRS and Cassini's VIMS from about 5 to 8 microns; this wavelength regime is not accessible to ground-based telescopes due to terrestrial atmospheric contamination. JWST will 
be able to observe near only one phase angle (i.e., near zero). This could be a strength, however, as CIRS data analysis is currently hampered by the need to disentangle too many changing parameters.

2006 September 3, VLTNISIR 18.7- $\mu$ m image
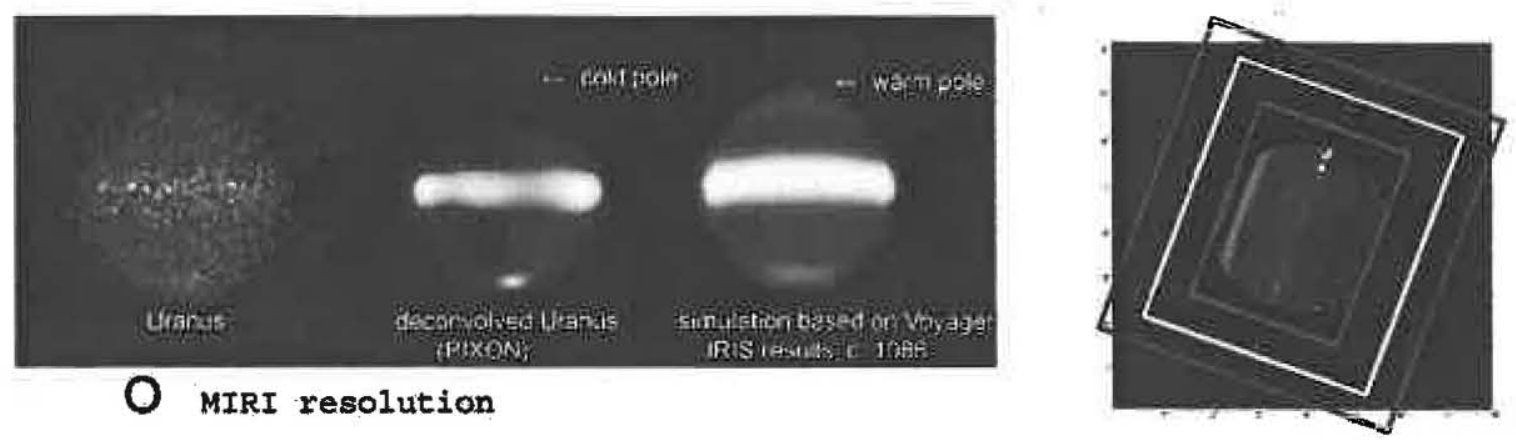

Figure 5. On the left is a 18.7 micron image of Uranus by Orton et al. taken with the VLT. For comparison, I show a simulated MIRI image of Uranus based on the Voyager observations, comparing a cold pole and warm pole. On the right, Uranus fits well within the field of view of the MIRI integral field spectroscopy.

At visible and near-infrared wavelengths, JWST cannot compete with Cassini. However, if the Cassini mission ends as scheduled in 2017, JWST will extend temporal coverage of the rings in the post-Cassini era. Imaging spatial resolution should be good enough to study ring spokes, an ephemeral phenomenon that is at maximum near equinox. Saturn's next equinox is in 2024, 6 years after JWST's 2018 launch, and thus within the extended JWST mission phase. Saturn's faint rings are also best observed near equinox.

\section{REFERENCES}

[1] Agol, E., Gogarten, S. M., Gorjian, V., \& Kimball, A. 2009, ApJ, 697, 1010

[2] Benecchi, S. D., Noll, K. S., Grundy, W. M., Buie, M. W., Stephens, D. C., \& Levison, H. F. 2009, Icarus, $200,292$.

[3] Charbonneau et al 2007, ApJ, 658, 1322

[4] Clampin, M., 2009, ASTRO2010, White Paper number 46: Comparative Planetology: Transiting Exoplanet Science with JWST

[5] Deming, D., et al 2009, PASP, 121, 952

[6] Gardner, J. P., et al 2006, SSRv, 123, 485

[7] Gardner, J. P., Stiavelli, M., \& Mather, J. C. 2010, White Paper: James Webb Space Telescope Studies of Dark Energy

[8] Gehrels, N., et al. 2009, Report on the Science Coordination Group Activities for the Joint Dark Energy Mission (JDEM)

[9] Grogin, N., et al. 2011, ApJS, 197, 35

[10]Humphreys, E. M. L., Reid, M. J., Greenhill, L. J., Moran, J. M., \& Argon, A. L., 2008, ApJ, 672, 800.

[11] Jarosik, N., et al. 2011, ApJS, 192, 14

[12] Knutson et al 2007, Nature, 447, 183

[13] Kravtsov, A. V., arXiv:0906.3295, Advances in Astronomy, 2010, 281913

[14]Lunine, J., et al 2010, White Paper, JWST Planetary Observations within the Solar System

[15]Marshall, P. J., et al. 2010, BAAS, 215, 401.15, vol. 41, p. 219

[16] Massey, R., et al. 2007, MNRAS, 376, 13

[17] Perlmutter, S., et al. 1999, ApJ, 517, 565

[18] Ricker, G., et al 2010, AAS, 215, 450.06

[19] Riess, A., et al 1998, AJ, 116, 1009

[20] Riess, A., et al 2001, ApJ, 560, 49

[21]Riess, A., et al 2007, ApJ, 659, 98

[22] Riess, A., et al 2011, ApJ, 730, 119 
[23] Seager, S., Deming, D., \& Válenti, J. A. 2009, arXiv:0808.1913

[24] Schrabback, T., et al 2007, A\&A, 516, 63

[25]Suyu, S. H., et al 2010, ApJ, 711, 201

[26] Swain, Mark R., Vasisht, G. \& Tinetti, G., Nature, 452, 329

[27] Thronson, H., Stiavelli, M., \& Tielens, A. [Astrophysics in the Next Decade], Springer, Netherlands, (2009)

[28] Treu, T. 2010, ARA\&A, 48, 87 